\title{
Spheroid three-dimensional culture enhances Notch signaling in cardiac progenitor cells
}

\begin{abstract}
Arianna Maurettit, Department of Biomedical Engineering, Eindhoven University of Technology, P0 Box 513, 5600 MB, Eindhoven, The Netherlands; Institute for Complex Molecular Systems (ICMS), Eindhoven University of Technology, Eindhoven, The Netherlands

Fabrizio Rossit, Department of Molecular Medicine, "Sapienza" University of Rome, Piazzale Aldo Moro 5, 00185 Rome, Italy

Noortje A. M. Bax, Department of Biomedical Engineering, Eindhoven University of Technology, PO Box 513, 5600 MB, Eindhoven, The Netherlands; Institute for Complex Molecular Systems (ICMS), Eindhoven University of Technology, Eindhoven, The Netherlands

Carmen Miano, Department of Molecular Medicine, "Sapienza" University of Rome, Piazzale Aldo Moro 5, 00185 Rome, Italy

Fabio Miraldi, Dipartimento di Scienze Cardiovascolari, Respiratorie, Nefrologiche e Geriatriche, "Sapienza" University of Rome, Viale del Policlinico 155, 00161 Rome, Italy

Marie José Goumans, Department of Molecular Cell Biology, Leiden University Medical Center, 2300 RC Leiden, The Netherlands

Elisa Messina, Department of Molecular Medicine, "Sapienza" University of Rome, Piazzale Aldo Moro 5, 00185 Rome, Italy; Department of Pediatrics and Pediatric Neuropsychiatry, "Sapienza" University of Rome, Viale Regina Elena 324, 00161 Rome, Italy

Alessandro Giacomello, Department of Molecular Medicine, "Sapienza" University of Rome, Piazzale Aldo Moro 5, 00185 Rome, Italy

Carlijn V. C. Bouten, Department of Biomedical Engineering, Eindhoven University of Technology, P0 Box 513, 5600 MB, Eindhoven, The Netherlands; Institute for Complex Molecular Systems (ICMS), Eindhoven University of Technology, Eindhoven, The Netherlands

Cecilia Sahlgren, Department of Biomedical Engineering, Eindhoven University of Technology, PO Box 513, 5600 MB, Eindhoven, The Netherlands; Institute for Complex Molecular Systems (ICMS), Eindhoven University of Technology, Eindhoven, The Netherlands; Cell biology, Faculty of Science and Engineering, Åbo Akademi University, FI-20520 Turku, Finland; Turku Centre for Biotechnology, University of Turku and Åbo Akademi University, Fl-20520 Turku, Finland
\end{abstract}

Address all correspondence to Arianna Mauretti at a.mauretti@tue.nl

(Received 13 April 2017; accepted 21 August 2017)

\begin{abstract}
Cardiac progenitor cells (CPCs) are a promising candidate for cardiac regeneration, and the interaction between CPCs and their microenvironment can influence their regenerative response. Notch signaling plays a key role in cell fate decisions in the developing and adult heart. Here, we investigated the effect of three-dimensional (3D) spheroid culture, as a model of the 3D microenvironment, on Notch in fetal and adult human CPCs, under room air (20\%) and physiological (5\%) oxygen tension. Notch signaling is enhanced in 3D spheroids; spheroid culture under $5 \% \mathrm{O}_{2}$ further increases Notch signaling enhancement, and might ultimately improve the regenerative potential of CPCs.
\end{abstract}

\section{Introduction}

Cardiac disease is one of the main causes of death worldwide. After injury, the heart muscle displays very limited regeneration, in which resident cardiac progenitor cells (CPCs) seem to play a role. ${ }^{[1]}$ Despite their acknowledged regenerative potential, ${ }^{[2-4]}$ very little is known about the actual role of CPCs, particularly of human origin, in cardiac repair and regeneration. Among the different CPC populations that have been identified, human cardiomyocyte progenitor cells (CMPCs) represent a promising candidate for cardiac regeneration, due to their presence in fetal and adult heart, and the capacity to differentiate into the three cardiac lineages (cardiomyocytes, endothelial, and smooth muscle cells). ${ }^{[3,5]}$ Another interesting source of human CPCs is represented by the cardiosphere model. Cardiospheres (CSps) are cardiac-derived multicellular

$\dagger$ These authors contributed equally to this work. spheroids, spontaneously formed by cells migrating from cardiac tissue explants and subsequently cultured on a poly-D-lysine-coated dish. ${ }^{[6,7]} \mathrm{CSps}$ can serve as an interesting in vitro model of the cardiac stem cell niche. ${ }^{[8]}$ Compared with traditional two-dimensional (2D) culture, multicellular spheroids provide a microenvironment that more closely mimics the in vivo physiology. These model systems recapitulate key features of the endogenous cell niche, such as improved cellcell and cell-extracellular matrix (ECM) contacts, as well as gradients of oxygen and nutrients between periphery and core of the spheroid. ${ }^{[8-11]}$ Improved knowledge on the mechanisms that regulate $\mathrm{CPC}$ behavior and functions, such as survival, proliferation, and differentiation, is needed. In particular, the interaction of CPCs with the niche in which they reside is of pivotal importance to ultimately direct their behavior toward cardiac regeneration.

Notch is a cell-cell signaling pathway that regulates many cellular functions, such as survival, proliferation, and 
differentiation. ${ }^{[12-14]}$ In the heart, Notch is essential during development, ${ }^{[15,16]}$ and it is inactivated in cardiomyocytes after birth. ${ }^{[14]}$ However, its reactivation after myocardial damage indicates that Notch plays a key role in cardiac repair. ${ }^{[13,17]}$ In CPCs, activation of the Notch pathway is known to induce cell proliferation, ${ }^{[13]}$ and is needed for the onset of cardiomyogenic differentiation, at the same time maintaining the cells in a proliferative state. $^{[13]}$

Here we use CSps, of both adult (CSps) and fetal origin (CMPC-CSps), as a model to investigate the Notch pathway in CPCs under room air $\left(20 \% \mathrm{O}_{2}\right)$ and tissue physiological $\left(5 \% \mathrm{O}_{2}\right)$ oxygen tension (often referred to as "hypoxia"). Our results show that a 3D in-vivo-like microenvironment, as provided by the CSp system, enhances Notch signaling in fetal and adult CPCs as compared with the respective cell monolayers. Moreover, the signaling enhancement provided by the 3D culture is further increased under hypoxic conditions. These findings provide fundamental knowledge on the interactions between CPCs and the conditions of the cardiac microenvironment, such as 3D culture and different oxygen tension conditions.

\section{Materials and methods}

CMPCs were isolated from fetal human hearts by magnetic sorting using Sca-1-coupled beads. L9TB cell line was immortalized by lentiviral transduction of hTert and BMI-1, and cultured in $\mathrm{SP}++$ growth medium, according to published protocols. ${ }^{[5]}$ CMPC-CSps were obtained by seeding L9TB CMPCs on poly-D-lysine-coated 12-multiwell plates with a density of $1.6 \times 10^{4}$ cells $/ \mathrm{cm}^{2}$ and cultured in CSp medium (CSM). Adult explant-derived cells (EDCs) were isolated from human surgical auricular biopsies, as previously described. ${ }^{[6,7]}$ Once $80 \%$ of confluency was reached, EDCs were collected and seeded on poly-D-lysine-coated 12-multiwell plates with a cell density of $9 \times 10^{3}$ cells $/ \mathrm{cm}^{2}$ to form CSps. CMPC-CSps and CSps spontaneously formed after 5 days of culture in either room air $\left(20 \% \mathrm{O}_{2}\right)$ or physiologic $\left(5 \% \mathrm{O}_{2}\right)$ oxygen conditions, and collected at this time-point. CSps were seeded on a fibronectin-coated flask to obtain CSp derived cells (CDCs). CMPCs, CMPC-CSps, EDCs, CSps, and CDCs were grown in either room air $\left(20 \% \mathrm{O}_{2}\right)$ or in tissue physiologic $\left(5 \% \mathrm{O}_{2}\right)$ oxygen conditions Table I; for details on cell isolation methods and culture medium composition; see Supplementary Material).

Table I. Schematic summary of all cell type and culture conditions used in this article.

\begin{tabular}{|c|c|c|c|}
\hline Abbreviation & Origin & \multicolumn{2}{|c|}{ Culture condition } \\
\hline CMPCs & Fetal & Monolayer (2D) & $5 \% / 20 \% \mathrm{O}_{2}$ \\
\hline CMPC-CSps & & Spheroid (3D) & $5 \% / 20 \% \mathrm{O}_{2}$ \\
\hline EDCs & Adult & Monolayer (2D) & $5 \% / 20 \% \mathrm{O}_{2}$ \\
\hline CSps & & Spheroid (3D) & $5 \% / 20 \% \mathrm{O}_{2}$ \\
\hline CDCs & & Monolayer (2D) & $5 \% / 20 \% \mathrm{O}_{2}$ \\
\hline
\end{tabular}

Total RNA was isolated from one sample per group per experiment ( $n=4$ for fetal CPCs; $n=3-7$ for adult CPCs) using the Qiagen RNAeasy isolation kit according to the manufacturer's instructions (Qiagen). cDNA was synthesized with $250 \mathrm{ng}$ RNA per sample using M-MLV Reverse Transcriptase (Bio-Rad) and subjected to qPCR using iQTM SYBR@Green Supermix and the CFX384 detection system (Bio-Rad). Primer sequences for qPCR and annealing temperatures are presented in Table II.

For immunofluorescence labeling, all samples were washed with PBS (phosphate-buffered saline) at $37^{\circ} \mathrm{C}$, fixed in $3.7 \%$ (v/v) formaldehyde for $15 \mathrm{~min}$, and rinsed four times with washing buffer (see Supplementary Material). A blocking step in $1 \%$ horse serum was followed by staining with primary and secondary antibodies, and 4,,6-diamidino-2-phenylindole (DAPI) for nuclear staining. Used antibodies and dyes are listed in Table III. Immunofluorescence images were acquired with a Leica TCS SP5X confocal microscope.

All data are presented as mean \pm standard deviation (SD). For qPCR data analysis, cycle-threshold $\left(C_{\mathrm{t}}\right)$ values were normalized to the reference gene (GAPDH) value, and signals of CMPC-CSps, CSps, and CDCs were compared with those of the cell monolayer (CMPCs and EDCs for fetal and adult cells, respectively) and presented as fold change $\left(2^{-\Delta \Delta C t}\right)$. A list of $C_{\mathrm{t}}$ values of fetal and adult cells is presented in Supplementary Tables SI and SII, respectively. Statistical analysis was performed with Graphpad Prism 5.04. Student's $t$-test or Kruskal-Wallis test with Dunn's post hoc for multiple comparisons were performed where appropriate. Significance was assumed for $P<0.05$.

\section{Results}

Notch signaling is enhanced in CPC spheroids The effect of 3D culture on Notch signaling in fetal (CMPC-CSps) and adult (CSps) CPCs was evaluated by

Table II. List of sequences and annealing temperatures $\left(T_{\mathrm{a}}\right)$ of used primers (Fw: forward; Rv: reverse).

\begin{tabular}{llr}
\hline Gene & \multicolumn{1}{c}{ Primer sequence } & \multicolumn{1}{c}{$T_{\mathrm{a}}$} \\
\hline Notch 1 (N1) & Fw: CCTGAAGAACGGGGCTAACA & 60 \\
\cline { 2 - 2 } & Rv: GATGTCCCGGTTGGCAAAGT & \\
\hline Notch 2 (N2) & Fw: GGCTATGAATGCCAGTGTGC & 60 \\
\cline { 2 - 2 } & Rv: GGATCGGGGTCACAGTTGTC & \\
\hline Notch 3 (N3) & Fw: GCCATGCTGATGTCAATGCT & 60 \\
\cline { 2 - 2 } & Rv: CAGCCCAGTGTAAGGCTGAT & \\
\hline Jagged 1 (Jag1) & Fw: AATGGCTACCGGTGTGTCTG & \\
\cline { 2 - 2 } & Rv: CCCATGGTGATGCAAGGTCT & \\
\hline Hey1 & Fw: CGAAATCCCAAACTCCGATA & \\
\cline { 2 - 3 } & Rv: TGGATCACCTGAAATGCTG & \\
\hline
\end{tabular}


Table III. List of used antibodies and dyes.

\begin{tabular}{|c|c|c|c|c|c|c|}
\hline Antigen & Source & Cat. no & Isotype & Species & Label & Dilution \\
\hline Notch 1 (N1) & Santa Cruz & sc-6014 & $\lg G$ & Goat & - & $1 / 50$ \\
\hline Notch 2 (N2) & Santa Cruz & sc-5545 & $\lg G$ & Rabbit & - & $1 / 50$ \\
\hline Notch 3 (N3) & Santa Cruz & sc-7424 & $\lg G$ & Goat & - & $1 / 50$ \\
\hline Rabbit IgG & Molecular Probes & A21428 & $\lg G(H+L)$ & Goat & Alexa Fluor 555 & $1 / 200$ \\
\hline Goat IgG & Molecular Probes & A21432 & $\lg G(H+L)$ & Donkey & Alexa Fluor 555 & $1 / 200$ \\
\hline DAPI & Sigma-Aldrich & D-9542 & - & - & - & $1 / 500$ \\
\hline
\end{tabular}

analyzing the gene expression of Notch receptors Notch 1 (N1), Notch 2 (N2), and Notch 3 (N3), Notch ligand Jagged 1 (Jag1), and target gene Hey1, as compared with the respective cell monolayer from which the CSps derive (CMPCs and EDCs for fetal and adult, respectively). Under $20 \% \quad \mathrm{O}_{2}$, CMPC-CSps and CMPC monolayer showed a similar expression of N1. However, CMPC-CSps displayed increased expression of N2 and N3 (2.0- and 2.5-folds), and Jag1 (1.9-fold) [Fig. 1(a)], and a 13.8-fold upregulation of the target gene Hey1 [Fig. 1(b)].

Adult CSps displayed upregulation of N1 and N3 (2.2- and 2.9-folds, respectively) as compared with both adult cell monolayers (EDCs and CDCs), whereas N2 and Jag1 showed similar gene expression levels in all samples, although a modest downregulation was shown in CDCs as compared with CSps
[Fig. 1(c)]. Significant upregulation of Heyl was observed in CSps as compared with both EDCs and CDCs [6.6-fold change; Fig. 1(d)].

Immunofluorescent staining revealed the presence of the $\mathrm{N} 2$ receptor in the cell nuclei in CMPC-CSps as well as in the CMPC monolayers, supporting the activation of Notch signaling suggested by the gene expression data [Fig. 1(e, f)]. Similar protein localization was observed in adult CSps and cell monolayers (EDCs, CDCs; Supplementary Fig. S2). Staining for N1 and N3 showed localization to the cell membrane and around the cell nuclei, respectively (Supplementary Fig. S1).

These results indicate that spheroid $3 \mathrm{D}$ culture favors the activation of Notch signaling in CPCs, more evidently in adult CSps than in fetal CMPC-CSps.

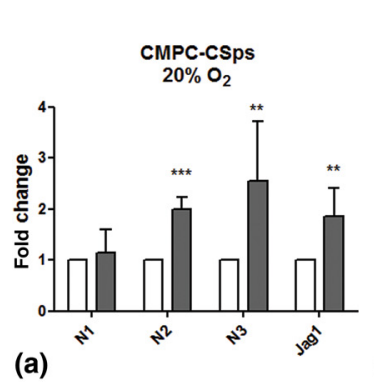

(a)

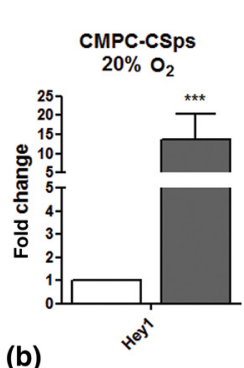

(b)

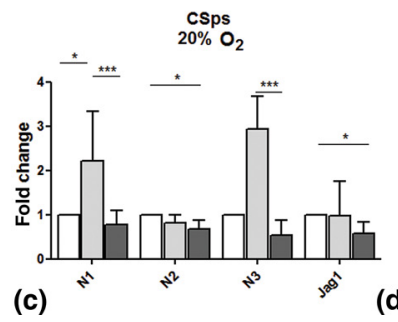

(d)

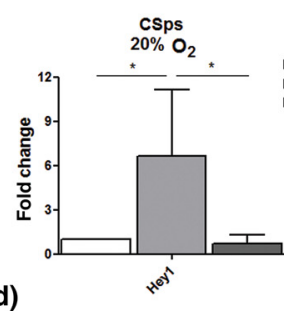

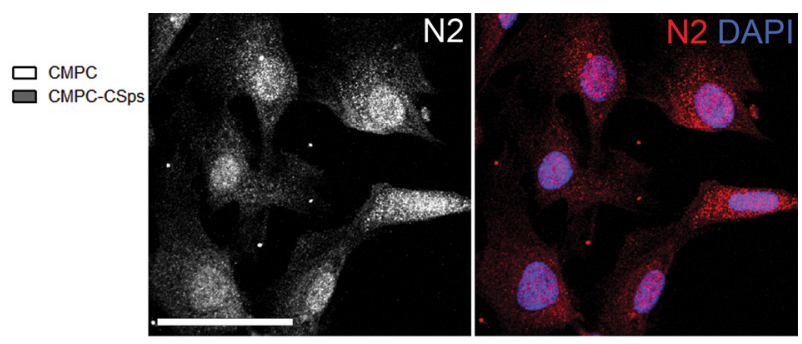

(e)

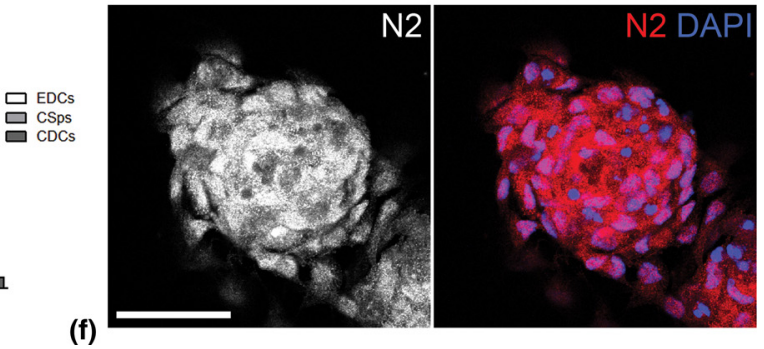

Figure 1. Notch signaling is enhanced by 3D spheroid culture in human CPCs. (a-d) Gene expression of Notch components (N1, N2, N3, Jag1) and target gene (Hey1) in (a, b) fetal CMPC-CSps $(n=4)$ and (c, d) adult CSps $(n=3-7)$ cultured under 20\% oxygen. (e, f) Immunofluorescent staining for Notch 2 (N2) in CMPCs (e) and CMPC-CSps (f) cultured at $20 \%$ oxygen. Nuclei are stained with DAPI. Scale bar $50 \mu \mathrm{m}$. Statistical test: Student's t-test for CMPC-CSps, KruskalWallis test followed by Dunn's post-test for adult CSps. ${ }^{\star} P<0.05,{ }^{*} P<0.01 ;{ }^{* \star}{ }^{\star} P<0.001$. 


\section{Notch signaling enhancement in CPC spheroids is increased by physiologic oxygen culture}

Oxygen tension is known to regulate many cell functions via the hypoxia and the Notch pathways, and is a key player in the stem cell niche. ${ }^{[18-20]}$ Therefore, we examined Notch signaling in fetal and adult CSps under physiological oxygen conditions $\left(5 \% \mathrm{O}_{2}\right)$. CMPC-CSps exhibited an increase in the RNA expression of all Notch components [Fig. 2(a)], and a remarkable upregulation of the target gene Heyl was observed [46.8-fold; Fig. 2(b)].

Similarly, adult CSps showed upregulation of N1 and N3 markers (2.3- and 2.2-folds, respectively) as compared with the monolayers (EDCs and CDCs), despite a slight downregulation of Jag1 (0.5-fold). No differences in the expression of N2 were observed between the three cell types [Fig. 2(c)]. Activation of the Notch downstream targets in adult CSps was demonstrated by Hey1 upregulation [22.7-fold; Fig. 2(d)].

Immunofluorescence staining showed the presence of N2 in the cell nuclei in CMPC-CSps and in the respective cell monolayer [Fig. 2(e, f)].

To determine the effect of hypoxia on Notch signaling in each cell type (adult and fetal CPCs) and culture condition (monolayer, CSps), we evaluated the expression of Notch markers at $5 \% \mathrm{O}_{2}$ as compared with $20 \% \mathrm{O}_{2}$. In fetal CMPCs and CMPC-CSps, a general downregulation of all markers was observed [Supplementary Fig. S3(a, b)], except for a 2.1-fold upregulation of Hey1 in CMPC-CSps at 5\% $\mathrm{O}_{2}$. In adult cells (CDCs, CSps), no changes were observed at $5 \%$ oxygen, except for a modest increase in the expression of $\mathrm{N} 1$ in CDCs (1.5-fold) and of Jag1 in CSps (1.5-fold) [Supplementary Fig. S3(c, d)].

The presented results indicate that the enhanced expression of Notch components and target genes in fetal and adult 3D CSps is further improved by culture under physiological oxygen tension.

\section{Discussion and outlook}

Multicellular spheroids, such as CSps, represent an in vitro model of the cardiac niche. ${ }^{[8,21]}$ The $3 \mathrm{D}$ environment and enhanced cell-cell and cell-ECM contacts offered by CSps can stimulate several molecular signaling and cellular functions. Notch is a highly conserved pathway that plays a key role in determining cell fate decisions in the heart. In CPCs, activation of Notch is needed to initiate cardiomyogenic differentiation; ${ }^{[13]}$ furthermore, transplantation of CPCs with increased Notch activity results in decreased infarct area and improved functions in an infarcted heart model. ${ }^{[22]}$ Previous studies have shown upregulation of Notch receptors 1 and 3 in CSps, as compared with the respective cell monolayer. ${ }^{[23]}$ Here, the upregulation of Notch receptors, and more importantly of the target gene Heyl (13.8- and 6.6-folds in fetal and adult cells, respectively), suggests activation of Notch pathway in fetal and adult CPCs cultured as CSps. Moreover, in the presence of a physiological oxygen condition (5\%), the 3D culture seemed to lead to an even stronger activation of Notch, as indicated by the enhanced upregulation of Heyl (46.8- and 22.7-folds in fetal and adult CPCs, respectively).

Interestingly, in CPC monolayers grown under $5 \% \mathrm{O}_{2}$ the expression of Notch components and target genes is either

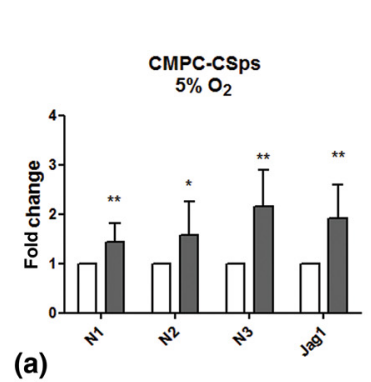

(a)

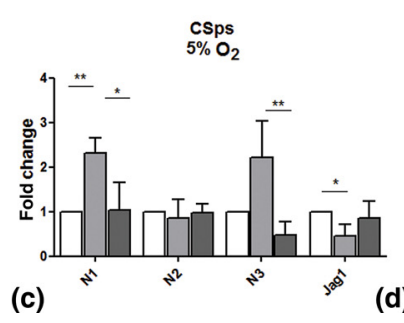

(d)

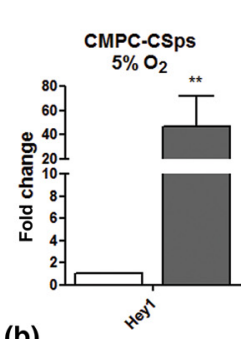

(b)

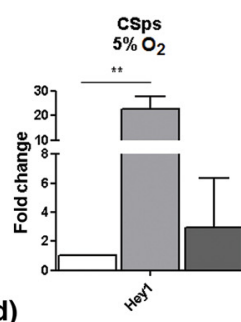

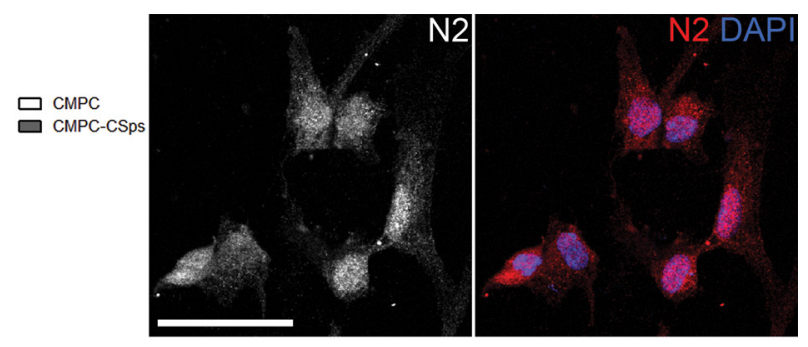

(e)

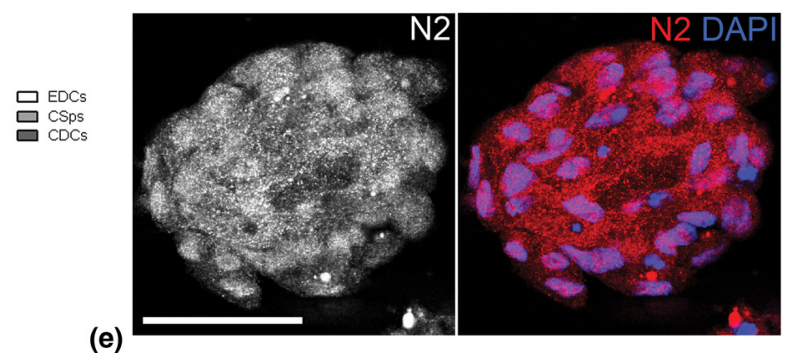

Figure 2. Notch signaling enhancement by 3D spheroid culture is increased by combination with physiological oxygen conditions in human CPCs. (a-d) Gene expression of Notch components (N1, N2, N3, Jag1) and target gene (Hey1) in (a, b) fetal CMPC-CSps $(n=4)$ and (c, d) adult CSps $(n=3-7)$ cultured under $5 \%$ oxygen. (e, f) Immunofluorescent staining for Notch 2 (N2) in CMPCs (e) and CMPC-CSps (f) cultured at 5\% oxygen. Nuclei are stained with DAPI. Scale bar 50 $\mu \mathrm{m}$. Statistical test: Student's $t$-test for CMPC-CSps, Kruskal-Wallis test followed by Dunn's post-test for adult CSps. ${ }^{*} P<0.05,{ }^{* \star} P<0.01 ;{ }^{* * *} P<0.001$. 


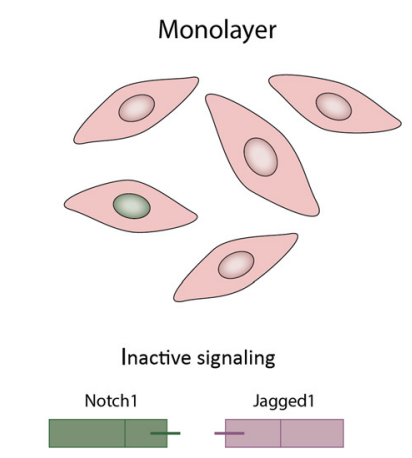

(a)

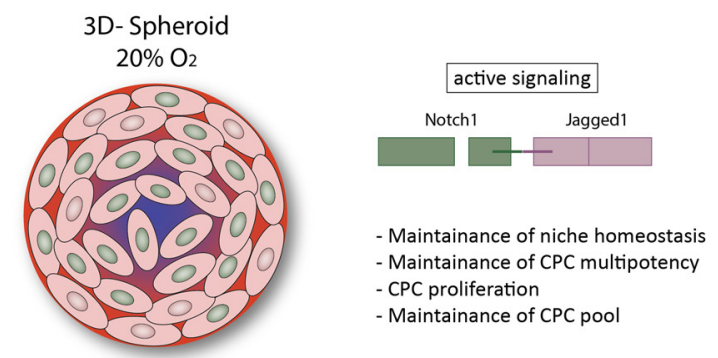

(b)
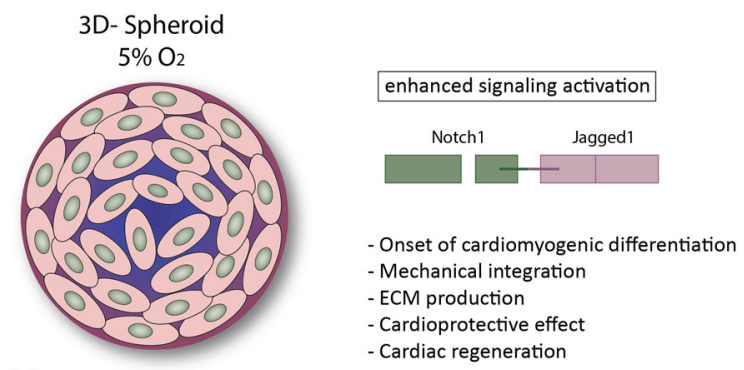

(c)

\author{
3D spheroid culture \\ - Increased cell-cell contacts \\ - Increased cell-ECM contacts \\ - Oxygen and nutrient gradients
}

Figure 3. Hypothesized putative mechanisms triggered by environmental conditions in CPCs. (a) In the cell monolayer, Notch activity is very low; (b, c) 3D spheroid culture provides improved cell-cell and cell-ECM contact and gradients of oxygen and nutrients, ${ }^{[9,26]}$ more similar to the in vivo environment. This results in enhanced Notch activation and might thus lead to: (b) CPC proliferation and maintenance of the stem cell pool and of niche homeostasis $\left(20 \% \mathrm{O}_{2}\right)$; or (c) cardiac differentiation, increased ECM production and modulation, mechanical integration and ultimately to a cardioprotective effect and cardiac regeneration $\left(\begin{array}{ll}5 \% & 0_{2}\end{array}\right)$.

decreased (fetal CPCs) or unchanged (adult CPCs) as compared with the $20 \% \mathrm{O}_{2}$ culture (Supplementary Fig. S3). However, when CPCs are grown as CSps under $5 \% \mathrm{O}_{2}$, the enhancement of Notch signaling is even stronger as compared with $20 \% \mathrm{O}_{2}$, suggesting that the $3 \mathrm{D}$ culture is needed for activation of Notch signaling increased under physiological oxygen tension in CPCs. In particular the reported data suggest that, in both fetal and adult CPCs, N2 is the component that translocates to the cell nuclei in response to spheroid formation as a result of Notch signaling activation. However, unlike fetal CPCs where all Notch markers are upregulated by the 3D culture, in adult CSps the gene expression of $\mathrm{N} 2$ seems unvaried as compared with the cell monolayer, indicating that in adult CPCs the activation of Notch is not due to increased RNA levels. The observation might be explained by the different developmental stage of the two cell types, and a distinct response to environmental factors on Notch signaling in fetal and adult cells.

Altogether, our findings suggest that in vivo-like conditions, as provided by the 3D spheroid system, particularly under with physiologic oxygen tension, enhance Notch signaling in human CPCs, of both fetal and adult origin. Given the role of Notch in
CPCs, this might be an important mechanism for several cell aspects of cardiac regeneration, such as maintenance of the CPC pool via enhanced survival and proliferation, maintenance of CPC multipotency and tissue homeostasis. However, it is tempting to speculate that a further increased activation of Notch (due to lower oxygen concentrations) might induce differentiation of CPCs into the cardiomyogenic lineage, resulting in improved extracellular matrix production and remodeling, ${ }^{[24]}$ mechanical integration, ${ }^{[25]}$ and might ultimately favor cardiac regeneration (Fig. 3). Further studies are needed in order to better understand the influence of microenvironmental conditions on human CPCs, and disclose their full potential for cardiac regeneration.

\section{Supplementary material}

The supplementary material for this article can be found at https://doi.org/10.1557/mrc.2017.82

\section{Acknowledgments}

This research was funded by the People Program (Marie Curie Actions) of the European Union's Seventh Framework Program FP7-People-2012-ITN “TECAS”, under grant agreement 
n. 317512 and the FP7-PEOPLE-2013-CIG "Stressfate", under grant agreement n. 618623. Dr. ir. Mark van Turnhout (Eindhoven University of Technology) is thankfully acknowledged for his help with confocal image analysis.

\section{References}

1. M.Y. Emmert, L.S. Emmert, A. Martens, I. Ismail, I. Schmidt-Richter, A. Gawol, B. Seifert, A. Haverich, U. Martin, and I. Gruh: Higher frequencies of BCRP+ cardiac resident cells in ischaemic human myocardium. Eur. Heart J. 34, 2830-2838 (2013).

2. P.P. Zwetsloot, A.M.D. Végh, S.J. Jansen Of Lorkeers, G.P.J. Van Hout, G. L. Currie, E.S. Sena, H. Gremmels, J.W. Buikema, M.J. Goumans, M. R. Macleod, P.A. Doevendans, S.A.J. Chamuleau, and J.P.G. Sluijter: Cardiac stem cell treatment in myocardial infarction: a systematic review and meta-analysis of preclinical studies. Circ. Res. 118, 1223-1232 (2016).

3. P. van Vliet, M.-J. Goumans, P. A. Doevendans, and J.P.G. Sluijter: Human cardiomyocyte progenitor cells: a short history of nearly everything. J. Cell. Mol. Med. 16, 1669-1673 (2012).

4. S. Bollini, N. Smart, and P.R. Riley: Resident cardiac progenitor cells: at the heart of regeneration. J. Mol. Cell. Cardiol. 50, 296-303 (2011).

5. A.M. Smits, P. van Vliet, C.H. Metz, T. Korfage, J.P. Sluijter, P. Doevendans, and M.J. Goumans: Human cardiomyocyte progenitor cells differentiate into functional mature cardiomyocytes: an in vitro model for studying human cardiac physiology and pathophysiology. Nat. Protoc. 4, 232-243 (2009).

6. E. Messina, L. De Angelis, G. Frati, S. Morrone, S. Chimenti, F. Fiordaliso, M. Salio, M. Battaglia, M.V.G. Latronico, M. Coletta, E. Vivarelli, L. Frati, G. Cossu, and A. Giacomello: Isolation and expansion of adult cardiac stem cells from human and murine heart. Circ. Res. 95, 911-921 (2004).

7. I. Chimenti, R. Gaetani, L. Barile, E. Forte, V. Ionta, F. Angelini, G. Frati, E. Messina, and A. Giacomello: Isolation and expansion of adult cardiac stem/progenitor cells in the form of cardiospheres from human cardiac biopsies and murine hearts, in Somatic Stem Cells: Methods and Protocols, edited by S.R. Singh, (Humana Press, Totowa, NJ, 2012) vol. 879, pp. 327-338.

8. T.S. Li, K. Cheng, S.-T. Lee, S. Matsushita, D. Davis, K. Malliaras, Y. Zhang, N. Matsushita, R.R. Smith, and E. Marbán: Cardiospheres recapitulate a niche-like microenvironment rich in stemness and cell-matrix interactions, rationalizing their enhanced functional potency for myocardial repair. Stem Cells 28, 2088-2098 (2010).

9. J. Günter, P. Wolint, A. Bopp, J. Steiger, E. Cambria, S.P. Hoerstrup, and M.Y. Emmert: Microtissues in cardiovascular medicine: regenerative potential based on a 3D microenvironment. Stem Cells Int. 2016, 1-20 (2016).

10.F. Gattazzo, A. Urciuolo, and P. Bonaldo: Extracellular matrix: a dynamic microenvironment for stem cell niche. Biochim. Biophys. Acta - Gen. Subj. 1840, 2506-2519 (2014).

11. T.M. Achilli, J. Meyer, and J.R. Morgan: Advances in the formation, use and understanding of multi-cellular spheroids. Expert Opin. Biol. Ther. 12, 1347-1360 (2012).

12. G. Luxán, G. D’Amato, D. MacGrogan, and J.L. De La Pompa: Endocardial Notch signaling in cardiac development and disease. Circ. Res. 118, e1e18 (2016).

13. A. Boni, K. Urbanek, A. Nascimbene, T. Hosoda, H. Zheng, F. Delucchi, K. Amano, A. Gonzalez, S. Vitale, C. Ojaimi, R. Rizzi, R. Bolli, K. E. Yutzey, M. Rota, J. Kajstura, P. Anversa, and A. Leri: Notch1 regulates the fate of cardiac progenitor cells. Proc. Natl. Acad. Sci. U.S.A. 105, 15529-15534 (2008).

14. C. Collesi, L. Zentilin, G. Sinagra, and M. Giacca: Notch1 signaling stimulates proliferation of immature cardiomyocytes. J. Cell Biol. 183, 117128 (2008).

15. M. Nemir and T. Pedrazzini: Functional role of Notch signaling in the developing and postnatal heart. J. Mol. Cell. Cardiol. 45, 495-504 (2008).

16. J.L. De la Pompa and J.A. Epstein: Coordinating tissue interactions: Notch signaling in cardiac development and disease. Dev. Cell 22, 244-254 (2012).
17. P. Rizzo, D. Mele, C. Caliceti, M. Pannella, C. Fortini, A.G. Clementz, M. B. Morelli, G. Aquila, P. Ameri, and R. Ferrari: The role of notch in the cardiovascular system: potential adverse effects of investigational notch inhibitors. Front. Oncol. 4, 1-11 (2014).

18. H. Main, K.L. Lee, H. Yang, S. Haapa-Paananen, H. Edgren, S. Jin, C. Sahlgren, O. Kallioniemi, L. Poellinger, B. Lim, and U. Lendahl: Interactions between Notch- and hypoxia-induced transcriptomes in embryonic stem cells. Exp. Cell Res. 316, 1610-1624 (2010).

19. U. Lendahl, K.L. Lee, H. Yang, and L. Poellinger: Generating specificity and diversity in the transcriptional response to hypoxia. Nat. Rev. Genet. 10, 821-832 (2009).

20. M.V. Gustafsson, X. Zheng, T. Pereira, K. Gradin, S. Jin, J. Lundkvist, J. L. Ruas, L. Poellinger, U. Lendahl, and M. Bondesson: Hypoxia requires Notch signaling to maintain the undifferentiated cell state. Dev. Cell $\mathbf{9}$, 617-628 (2005).

21. D.R. Davis, Y. Zhang, R.R. Smith, K. Cheng, J. Terrovitis, K. Malliaras, T. S. Li, A. White, R. Makkar, and E. Marbàn: Validation of the cardiosphere method to culture cardiac progenitor cells from myocardial tissue. PLOS ONE 4, e7195 (2009).

22. N. Gude, E. Joyo, H. Toko, P. Quijada, M. Villanueva, N. Hariharan, V. Sacchi, S. Truffa, A. Joyo, M. Voelkers, R. Alvarez, and M. A. Sussman: Notch activation enhances lineage commitment and protective signaling in cardiac progenitor cells. Basic Res. Cardiol. 110 (2015). doi: $10.1007 / \mathrm{s} 00395-015-0488-3$

23. E. Forte, F. Miraldi, I. Chimenti, F. Angelini, A. Zeuner, A. Giacomello, M. Mercola, and E. Messina: TGF $\beta$-dependent epithelial-to-mesenchymal transition is required to generate cardiospheres from human adult heart biopsies. Stem Cells Dev.. 21, 3081-3090 (2012).

24. N. A. M. Bax, M.H. van Marion, B. Shah, M.-J. Goumans, C.V.C. Bouten, and D.W.J. van der Schaft: Matrix production and remodeling capacity of cardiomyocyte progenitor cells during in vitro differentiation. J. Mol. Cell. Cardiol. 53, 497-508 (2012).

25. A. Mauretti, N.A. M. Bax, M.H. van Marion, M.J. Goumans, C. Sahlgren, and C.V.C. Bouten: Cardiomyocyte progenitor cell mechanoresponse unrevealed: strain avoidance and mechanosome development. Integr. Biol. 8, 991-1001 (2016).

26. E. di Costanzo, A. Giacomello, E. Messina, R. Natalini, G. Pontrelli, F. Rossi, R. Smits, M. Twarogowska: A discrete in continuous mathematical model of cardiac progenitor cells formation and growth as spheroid clusters (Cardiospheres). Math. Med. Biol. 1-24 (2017). pii: dqw022, doi: 10.1093/imammb/dqw022 